\title{
Stability and boundedness of nonautonomous neutral differential equation with delay
}

\author{
Moussadek Remili*, Linda D. Oudjedi
}

Abstract. We consider the nonautonomous neutral differential equation with delay

$$
\begin{aligned}
& {\left[p(t)\left(q(t)\left(x(t)+\beta_{1} x\left(t-r_{1}\right)\right)^{\prime}\right)^{\prime}\right]^{\prime}+a(t)\left(x^{\prime \prime}(t)+\beta_{2} x^{\prime \prime}\left(t-r_{2}\right)\right)} \\
& +b(t)\left(x^{\prime}(t)+\beta_{3} x^{\prime}\left(t-r_{3}\right)\right)+c(t) f(x(t-\sigma))=e\left(t, x, x^{\prime}, x^{\prime \prime}\right) .
\end{aligned}
$$

Using the method of Lyapunov, we give conditions for the uniform asymptotic stability and uniform boundedness and square integrability of solutions for the considered system. Our theorems generalize and extend some related results known in the literature. Example is given to show our results.

\section{INTRODUCTION}

In our paper, we study the asymptotic uniform stability of the nonautonomous neutral differential equation with delay and coefficients of the form

$$
\begin{aligned}
& {\left[p(t)\left(q(t)\left(x(t)+\beta_{1} x\left(t-r_{1}\right)\right)^{\prime}\right)^{\prime}\right]^{\prime}+a(t)\left(x^{\prime \prime}(t)+\beta_{2} x^{\prime \prime}\left(t-r_{2}\right)\right)} \\
& +b(t)\left(x^{\prime}(t)+\beta_{3} x^{\prime}\left(t-r_{3}\right)\right)+c(t) f(x(t-\sigma))=0,
\end{aligned}
$$

and the boundedness and the square integrability of

$$
\begin{aligned}
& {\left[p(t)\left(q(t)\left(x(t)+\beta_{1} x\left(t-r_{1}\right)\right)^{\prime}\right)^{\prime}\right]^{\prime}+a(t)\left(x^{\prime \prime}(t)+\beta_{2} x^{\prime \prime}\left(t-r_{2}\right)\right)} \\
& +b(t)\left(x^{\prime}(t)+\beta_{3} x^{\prime}\left(t-r_{3}\right)\right)+c(t) f(x(t-\sigma))=e\left(t, x, x^{\prime}, x^{\prime \prime}\right),
\end{aligned}
$$

for all $t \geq t_{1}=t_{0}+\bar{r}$, where $\bar{r}=\sup \left\{\sigma, r_{i}\right\}, \beta_{i}$ are constants with $0 \leq \beta_{i} \leq 1$ and $\sigma, r_{i} \geq 0(\forall i=1,2,3)$.

$p(),. q(),. a(),. b(),. c(),. e($.$) and f($.$) are continuous functions depending only$

2010 Mathematics Subject Classification. Primary: 34D05; Secondary: 34D20; Thirdary: $34 \mathrm{~K} 40$.

Key words and phrases. Uniform ultimate boundedness, square integrability, Lyapunov functional, neutral differential equation of third order.

Full paper. Received 1 February 2019, revised 20 December 2019, accepted 25 December 2019, available online 29 February 2020.

*Corresponding Author. 
on the arguments shown. In addition, it is also supposed that the derivatives $p^{\prime}(t)$ and $q^{\prime \prime}(t)$ exist and are continuous.

By a solution of (2) we mean a continuous function $x:\left[t_{x}, \infty\right) \rightarrow \mathbb{R}$ such that $x(t)+\beta_{1} x\left(t-r_{1}\right) \in C^{3}\left(\left[t_{x}, \infty\right), \mathbb{R}\right)$ and which satisfies equation $(2)$ on $\left[t_{x}, \infty\right)$.

Several authors have investigated the uniform stability and boundedness of solutions of certain differential equations of the third order. We can mention in this direction, the works of Ayhan and Tunç [2], Graef et al. [10, 11], Mahmoud [16], Omeike [17], Oudjedi et al. [18], Remili et al. [19]-[30], where the second Lyapunov method was used. This problem for neutral differential equations has received considerable attention in recent years, Baculíková [3], Mihalíková and Kostiková [4], Das and Misra [5], Dorociaková [6], Došlá and Liška [7, 8], Kulenovic et al. [15], Tian et al. [31], Li et al. [32], Yu et al. [33], Yu Jianshe [34]. Many books dealt with the neutral delay differential equation and obtained many good results, for example Arino et al. [1], Hale [13, 14], El'sgol'ts [9].

However, to the best of our knowledge from the literature, by this time, no attention was given to the investigation of the uniformly asymptotic stability/ uniformly boundedness and square integrability in the systems of nonlinear neutral differential equations of the third order with delay, using the Lyapunov's direct (or second) method, except the recent work in 2018 of Graef et al. [12].

Besides, this paper may be useful for researchers working on the qualitative behaviors of solutions of third differential equations and completes that in the literature. These cases show the novelty and originality of the present paper.

\section{Asymptotic stability}

Suppose that there are positive constants $a_{0}, a_{1}, c_{0}, b_{1}, p_{0}, p_{1}, q_{0}, q_{1}, l, L, \delta$, $d, M, A$ and $B$ such that the following conditions are satisfied, $\forall t \geq t_{1}=$ $t_{0}+\bar{r}:$

$\left.\mathrm{J}_{0}\right) \quad 0<a_{0} \leq a(t) \leq a_{1}, \quad 0<c_{0} \leq c(t) \leq b(t) \leq b_{1}$,

$0<p_{0} \leq p(t) \leq p_{1}, \quad 0<q_{0} \leq q(t) \leq q_{1}$

J $\mathrm{J}_{1} 2 \delta p_{1} q_{1}<d<a_{0}, \quad-l \leq b^{\prime}(t) \leq c^{\prime}(t) \leq 0$, $-L \leq p^{\prime}(t) \leq 0, \quad-L \leq q^{\prime}(t) \leq 0$

$\left.\mathrm{J}_{2}\right) f(0)=0, \frac{f(x)}{x} \geq M>0(x \neq 0)$, and $\left|f^{\prime}(x)\right| \leq \delta$ for all $x$;

$\left.\mathrm{J}_{3}\right) d a^{\prime}(t)+2 c_{0}\left(p_{1} q_{1} \delta\left(1+\frac{\beta_{1}}{2}\right)-d\right)+\left(\beta_{2} a_{1}+\beta_{3} b_{1}\right)\left(d+L \beta_{1} p_{1}\right)$ $+\beta_{1} p_{1}\left(b_{1} q_{1} \delta+b_{1} L+b_{1} q_{1}\left(1+\beta_{1}\right)+L\left(a_{1}-d\right)\right)+b_{1} \beta_{3} n_{1} \leq-A<0 ;$

$\left.\mathrm{J}_{4}\right) p_{0} q_{0}\left(2-\beta_{1}\right)\left(d-a_{0}\right)+p_{1} q_{1}\left(\beta_{1} b_{1}+\beta_{2} a_{1}+\beta_{3} b_{1}\right)\left(1+\beta_{1}\right)$ $+p_{1}\left(a_{1}-d\right)\left(\beta_{1} q_{1}+L\left(1+\beta_{1}\right)\right)+a_{1} \beta_{2} n_{1} \leq-B<0$, 
where

$$
n_{1}=d+p_{1}\left(1+\beta_{1}\right)\left(L+q_{1}\right) .
$$

The equation (1) is equivalent to the following system

$$
\begin{aligned}
x^{\prime}= & y \\
y^{\prime}= & z \\
(p(t) Z)^{\prime}= & -a(t) z-\beta_{2} a(t) z\left(t-r_{2}\right)-b(t) y-\beta_{3} b(t) y\left(t-r_{3}\right) \\
& -c(t) f(x(t))+c(t) \int_{t-\sigma}^{t} f^{\prime}(x(s)) y(s) d s .
\end{aligned}
$$

For the brevity, we put

$$
Y(t)=q(t)\left(y(t)+\beta_{1} y\left(t-r_{1}\right)\right) .
$$

According to (3), we have

$$
\begin{aligned}
Y^{\prime}(t)=Z(t) & =\left[q(t)\left(y(t)+\beta_{1} y\left(t-r_{1}\right)\right)\right]^{\prime} \\
& =q^{\prime}(t)\left(y(t)+\beta_{1} y\left(t-r_{1}\right)\right)+q(t)\left(z(t)+\beta_{1} z\left(t-r_{1}\right)\right) .
\end{aligned}
$$

Theorem 1. Assume that all assuptions $\left(J_{0}-J_{4}\right)$ hold. Then, the zero solution of (3) is asymptotically stable if

$$
\sigma<\min \left\{\frac{A}{b_{1} \delta\left(2 d+p_{1}\left(2 L+q_{1}\right)\left(1+\beta_{1}\right)\right)}, \frac{B}{b_{1} p_{1} q_{1} \delta\left(1+\beta_{1}\right)}\right\} .
$$

Proof. Define a Lyapunov functional $W(t, x, y, z)$ such that $W(t, 0)=0$ and

$$
W=\exp \left(-\frac{1}{\omega} \int_{t_{1}}^{t}\left(\left|p^{\prime}(s)\right|+\left|q^{\prime}(s)\right|\right) d s\right) V
$$

where

$$
\begin{aligned}
V & =d c(t) F(x)+c(t) p(t) Y f(x)+\frac{b(t) p(t)}{2 q(t)} Y^{2}+\frac{1}{2} p^{2}(t) Z^{2} \\
& +d p(t) y Z+\frac{1}{2} d a(t) y^{2}+\Delta(t),
\end{aligned}
$$

such that

$$
\begin{aligned}
\Delta(t) & =\mu_{1} \int_{t-r_{1}}^{t} z^{2}(s) d s+\mu_{2} \int_{t-r_{2}}^{t} z^{2}(s) d s+\eta_{1} \int_{t-r_{1}}^{t} y^{2}(s) d s \\
& +\eta_{2} \int_{t-r_{3}}^{t} y^{2}(s) d s+\lambda \int_{-\sigma}^{0} \int_{t+s}^{t} y^{2}(\tau) d \tau d s
\end{aligned}
$$

and $F(x)=\int_{0}^{x} f(u) d u . \mu_{i}, \eta_{i}, \omega$ and $\lambda$ are to be selected below suitably. The functional $V$ defined in the equation (5) can be written in the form

$$
V=c(t) \int_{0}^{x}\left[d-2 p(t) q(t) f^{\prime}(u)\right] f(u) d u+\frac{c(t) p(t)}{q(t)}\left(\frac{1}{2} Y+q(t) f(x)\right)^{2}
$$




$$
+\frac{b(t) p(t)}{2 q(t)}\left(1-\frac{c(t)}{2 b(t)}\right) Y^{2}+\frac{1}{2}(p(t) Z+d y)^{2}+\frac{d}{2}(a(t)-d) y^{2}+\Delta(t) .
$$

Since

$$
\Delta(t) \geq 0
$$

by $\left(J_{0}\right)-\left(J_{2}\right)$, it follows that

$$
V \geq \frac{c_{0} M}{2}\left[d-2 p_{1} q_{1} \delta\right] x^{2}+\frac{c_{0} p_{0}}{4 q_{1}} Y^{2}+\frac{1}{2}(p(t) Z+d y)^{2}+\frac{d}{2}\left(a_{0}-d\right) y^{2} .
$$

Then, there exists $k>0$ such that

$$
V \geq k\left(x^{2}+y^{2}+Y^{2}+Z^{2}\right),
$$

by $\left(\mathrm{J}_{0}, \mathrm{~J}_{1}\right)$, we conclude that

$$
W \geq k_{0}\left(x^{2}+y^{2}+Y^{2}+Z^{2}\right), \quad k_{0}=k e^{\frac{1}{\omega}\left(p_{0}-p_{1}+q_{0}-q_{1}\right)} .
$$

The derivative of the functional $\mathrm{V}$ along the trajectories of the system is given by

$$
\begin{aligned}
V^{\prime} & =\psi_{0}(t)+\psi_{1}(t)+c(t) \psi_{2}(t)+\left[p(t) q(t)(d-a(t))+\mu_{1}+\mu_{2}\right] z^{2} \\
& +\left[\frac{d}{2} a^{\prime}(t)-d b(t)+c(t) p(t) q(t) f^{\prime}(x)+\eta_{1}+\eta_{2}+\lambda \sigma\right] y^{2}-\mu_{1} z^{2}\left(t-r_{1}\right) \\
& -\mu_{2} z^{2}\left(t-r_{2}\right)-\eta_{1} y^{2}\left(t-r_{1}\right)-\eta_{2} y^{2}\left(t-r_{3}\right)-\lambda \int_{t-\sigma}^{t} y^{2}(s) d s,
\end{aligned}
$$

where

$$
\psi_{0}(t)=d c^{\prime}(t) F(x)+(c(t) p(t))^{\prime} Y f(x)+\left(\frac{b(t) p(t)}{2 q(t)}\right)^{\prime} Y^{2},
$$

and

$$
\begin{aligned}
\psi_{1}(t) & =\beta_{1} c(t) p(t) q(t) f^{\prime}(x) y y\left(t-r_{1}\right)+\beta_{1} b(t) p(t) y\left(t-r_{1}\right) Z \\
& -p(t)\left(\beta_{2} a(t) z\left(t-r_{2}\right)+\beta_{3} b(t) y\left(t-r_{3}\right)\right)\left(Z+\frac{d}{p(t)} y\right) \\
& +p(t)(d-a(t)) z\left(q^{\prime}(t) y+\beta_{1} q^{\prime}(t) y\left(t-r_{1}\right)+\beta_{1} q(t) z\left(t-r_{1}\right)\right) \\
\psi_{2}(t) & =(p(t) Z+d y) \int_{t-\sigma}^{t} f^{\prime}(x(s)) y(s) d s .
\end{aligned}
$$

If $c^{\prime}(t)<0$, the quantity $\psi_{0}(t)$ can be written as,

$$
\begin{aligned}
\psi_{0}(t) & =c^{\prime}(t)\left(d F(x)+\frac{b^{\prime}(t) p(t)}{2 q(t) c^{\prime}(t)}\left\{Y+\frac{c^{\prime}(t) q(t)}{b^{\prime}(t)} f(x)\right\}^{2}\right. \\
& \left.-\frac{c^{\prime}(t) p(t) q(t)}{2 b^{\prime}(t)} f^{2}(x)\right)-\frac{b(t) p(t) q^{\prime}(t)}{2 q^{2}(t)} Y^{2}
\end{aligned}
$$




$$
+p^{\prime}(t)\left[\frac{b(t)}{2 q(t)}\left\{Y+\frac{c(t) q(t)}{b(t)} f(x)\right\}^{2}-\frac{c^{2}(t) q(t)}{2 b(t)} f^{2}(x)\right],
$$

by $\left(J_{0}\right)$ and $\left(J_{1}\right)$ we observe that

$$
0<\frac{c(t)}{b(t)} \leq 1 \quad \text { and } \quad 0 \leq \frac{c^{\prime}(t)}{b^{\prime}(t)} \leq 1
$$

thus

$$
\begin{aligned}
\psi_{0}(t) \leq & c^{\prime}(t)\left[\int_{0}^{x}\left(d-\delta p_{1} q_{1}\right) f(u) d u\right]-\frac{b(t) p(t) q^{\prime}(t)}{2 q^{2}(t)} Y^{2} \\
& -\frac{p^{\prime}(t) c(t) q(t)}{2} f^{2}(x) \\
\leq & \frac{b_{1} q_{1} \delta^{2}}{2}\left|p^{\prime}(t)\right| x^{2}+\frac{b_{1} p_{1}}{2 q_{0}^{2}}\left|q^{\prime}(t)\right| Y^{2} .
\end{aligned}
$$

If $c^{\prime}(t)=0$, then

$$
\begin{aligned}
\psi_{0}(t) & =c(t) p^{\prime}(t) Y f(x)+\left(\frac{b(t) p(t)}{2 q(t)}\right)^{\prime} Y^{2} \\
& =\frac{b^{\prime}(t) p(t)}{2 q(t)} Y^{2}+\frac{b(t) p^{\prime}(t)}{2 q(t)}\left[Y+\frac{c(t) q(t)}{b(t)} f(x)\right]^{2} \\
& -p^{\prime}(t) \frac{c^{2}(t) q(t)}{2 b(t)} f^{2}(x)-\frac{b(t) p(t) q^{\prime}(t)}{2 q^{2}(t)} Y^{2} \\
& \leq \frac{b_{1} q_{1} \delta^{2}}{2}\left|p^{\prime}(t)\right| x^{2}+\frac{b_{1} p_{1}}{2 q_{0}^{2}}\left|q^{\prime}(t)\right| Y^{2} .
\end{aligned}
$$

Hence, on combining (8) and (9), we have

$$
\psi_{0}(t) \leq \frac{b_{1} q_{1} \delta^{2}}{2}\left|p^{\prime}(t)\right| x^{2}+\frac{b_{1} p_{1}}{2 q_{0}^{2}}\left|q^{\prime}(t)\right| Y^{2}
$$

for all $t \geq t_{1}, x$ and $Y$.

From $\left(J_{0}\right),\left(J_{2}\right)$ and applying the estimate $2 u v \leq u^{2}+v^{2}$, it is not difficult to proof the following,

$$
\begin{aligned}
\psi_{1}(t) & \leq \frac{1}{2}\left(\beta_{1} b(t) p_{1} q_{1} \delta+p_{1}\left|q^{\prime}(t)\right|\left(\beta_{1} b_{1}+\beta_{2} a_{1}+\beta_{3} b_{1}+\left(a_{1}-d\right)\right)\right. \\
& \left.+d\left(\beta_{2} a_{1}+\beta_{3} b_{1}\right)\right) y^{2}+\frac{\beta_{1} p_{1}}{2}\left(b_{1} q_{1} \delta+b_{1} L\left(1+\beta_{3}\right)+b_{1} q_{1}\left(1+\beta_{1}\right)\right. \\
& \left.+\beta_{2} a_{1} L+L\left(a_{1}-d\right)\right) y^{2}\left(t-r_{1}\right)+\left(\frac { p _ { 1 } } { 2 } \left(\beta_{1} b_{1} q_{1}+\beta_{2} a_{1} q_{1}+\beta_{3} b_{1} q_{1}\right.\right. \\
& \left.\left.+L\left(a_{1}-d\right)\left(1+\beta_{1}\right)\right)+\frac{\beta_{1}}{2} p(t) q(t)(a(t)-d)\right) z^{2}+\frac{\beta_{1} p_{1} q_{1}}{2}\left(\beta_{1} b_{1}\right.
\end{aligned}
$$




$$
\begin{aligned}
& \left.+\beta_{2} a_{1}+\beta_{3} b_{1}+\left(a_{1}-d\right)\right) z^{2}\left(t-r_{1}\right)+\frac{\beta_{2} a_{1}}{2}\left(p_{1}\left(L+q_{1}\right)\left(1+\beta_{1}\right)\right. \\
& +d) z^{2}\left(t-r_{2}\right)+\frac{\beta_{3} b_{1}}{2}\left(p_{1}\left(L+q_{1}\right)\left(1+\beta_{1}\right)+d\right) y^{2}\left(t-r_{3}\right)
\end{aligned}
$$

and

$$
\begin{aligned}
\psi_{2}(t) \leq & \frac{\delta}{2}\left(\sigma \left[\left(d+p_{1} L\right) y^{2}+\beta_{1} p_{1} L y^{2}\left(t-r_{1}\right)+p_{1} q_{1} z^{2}+\right.\right. \\
& \left.\left.+\beta_{1} p_{1} q_{1} z^{2}\left(t-r_{1}\right)\right] n_{1} \int_{t-\sigma}^{t} y^{2}(s) d s\right) .
\end{aligned}
$$

By $\left(\mathrm{J}_{0}\right)$ and $\left(\mathrm{J}_{1}\right)$, we observe that $V^{\prime}$ can be replaced by

$$
\begin{aligned}
V^{\prime} \leq & \left(\frac{d}{2} a^{\prime}(t)+b(t)\left(p_{1} q_{1} \delta\left(1+\frac{\beta_{1}}{2}\right)-d\right)+\frac{d}{2}\left(\beta_{2} a_{1}+\beta_{3} b_{1}\right)+\eta_{1}+\eta_{2}\right. \\
+ & \left.\sigma\left(\lambda+\frac{b_{1} \delta}{2}\left(d+p_{1} L\right)\right)\right) y^{2}+\left(p_{0} q_{0}\left(d-a_{0}\right)\left(1-\frac{\beta_{1}}{2}\right)+\mu_{1}+\mu_{2}\right. \\
+ & \left.\frac{p_{1}}{2}\left(q_{1}\left(\beta_{1} b_{1}+\beta_{2} a_{1}+\beta_{3} b_{1}+b_{1} \delta \sigma\right)+L\left(a_{1}-d\right)\left(1+\beta_{1}\right)\right)\right) z^{2} \\
+ & \left(\frac { \beta _ { 1 } p _ { 1 } } { 2 } \left(b_{1} q_{1} \delta+b_{1} L\left(1+\beta_{3}\right)+b_{1} q_{1}\left(1+\beta_{1}\right)+\beta_{2} a_{1} L+L\left(a_{1}-d\right)\right.\right. \\
+ & \left.\left.b_{1} \delta \sigma L\right)-\eta_{1}\right) y^{2}\left(t-r_{1}\right)+\left(\frac { \beta _ { 1 } p _ { 1 } q _ { 1 } } { 2 } \left(\beta_{1} b_{1}+\beta_{2} a_{1}+\beta_{3} b_{1}\right.\right. \\
+ & \left.\left.\left(a_{1}-d\right)+b_{1} \delta \sigma\right)-\mu_{1}\right) z^{2}\left(t-r_{1}\right)+\psi_{4}(t)+\left(\frac{\beta_{2} a_{1}}{2} n_{1}-\mu_{2}\right) \\
& z^{2}\left(t-r_{2}\right)+\left(\frac{\beta_{3} b_{1}}{2} n_{1}-\eta_{2}\right) y^{2}\left(t-r_{3}\right)+\left(\frac{b_{1} \delta}{2} n_{1}-\lambda\right) \int_{t-\sigma}^{t} y^{2}(s) d s
\end{aligned}
$$

where

$$
\begin{aligned}
\psi_{4}(t) & =\frac{b_{1} q_{1} \delta^{2}}{2}\left|p^{\prime}(t)\right| x^{2}+\frac{p_{1}}{2}\left|q^{\prime}(t)\right|\left(\frac{b_{1}}{q_{0}^{2}} Y^{2}+\left(\beta_{1} b_{1}+\beta_{2} a_{1}+\beta_{3} b_{1}\right.\right. \\
& \left.\left.+\left(a_{1}-d\right)\right) y^{2}\right) \\
& \leq k_{1}\left(\left|p^{\prime}(t)\right|+\left|q^{\prime}(t)\right|\right)\left(x^{2}+y^{2}+Y^{2}\right),
\end{aligned}
$$

such that $k_{1}=\frac{1}{2} \max \left\{b_{1} q_{1} \delta^{2}, \frac{b_{1} p_{1}}{q_{0}^{2}}, p_{1}\left(\beta_{1} b_{1}+\beta_{2} a_{1}+\beta_{3} b_{1}+\left(a_{1}-d\right)\right)\right\}$.

Let

$$
\mu_{1}=\frac{\beta_{1} p_{1} q_{1}}{2}\left(\beta_{1} b_{1}+\beta_{2} a_{1}+\beta_{3} b_{1}+\left(a_{1}-d\right)+b_{1} \delta \sigma\right)
$$




$$
\begin{aligned}
& \eta_{1}=\frac{\beta_{1} p_{1}}{2}\left(b_{1} L\left(1+\beta_{3}\right)+b_{1} q_{1}\left(\delta+1+\beta_{1}\right)+L\left(a_{1}-d+\beta_{2} a_{1}+b_{1} \delta \sigma\right)\right) \\
& \mu_{2}=\frac{a_{1} \beta_{2}}{2} n_{1}, \quad \eta_{2}=\frac{b_{1} \beta_{3}}{2} n_{1}, \quad \lambda=\frac{b_{1} \delta}{2} n_{1} .
\end{aligned}
$$

Now, in view of estimates of $A, B$ and (6), this inequality becomes

$$
\begin{aligned}
V^{\prime} & \leq \frac{1}{2}\left(-A+\sigma b_{1} \delta\left(2 d+p_{1}\left(2 L+q_{1}\right)\left(1+\beta_{1}\right)\right)\right) y^{2}+\frac{k_{1}}{k}\left(\left|p^{\prime}(t)\right|+\left|q^{\prime}(t)\right|\right) V \\
& +\frac{1}{2}\left(-B+b_{1} p_{1} q_{1} \delta \sigma\left(1+\beta_{1}\right)\right) z^{2} .
\end{aligned}
$$

We take $\omega=\frac{k}{k_{1}}$, thus

$$
\begin{aligned}
W^{\prime} \leq & \frac{1}{2} \exp \left(-\frac{k_{1}}{k} \int_{0}^{t}\left(\left|p^{\prime}(s)\right|+\left|q^{\prime}(s)\right|\right) d s\right)\left(\left(-B+b_{1} p_{1} q_{1} \delta \sigma\left(1+\beta_{1}\right)\right) z^{2}\right. \\
& \left.+\left(-A+\sigma b_{1} \delta\left(2 d+p_{1}\left(2 L+q_{1}\right)\left(1+\beta_{1}\right)\right)\right) y^{2}\right)
\end{aligned}
$$

If

$$
\sigma<\min \left\{\frac{A}{b_{1} \delta\left(2 d+p_{1}\left(2 L+q_{1}\right)\left(1+\beta_{1}\right)\right)}, \frac{B}{b_{1} p_{1} q_{1} \delta\left(1+\beta_{1}\right)}\right\},
$$

then

$$
\frac{d}{d t} W(t, x, y, z) \leq-\delta_{1}\left(y^{2}+z^{2}\right), \text { for some } \delta_{1}>0 .
$$

Thus, all the conditions of theorem are satisfied. This shows that the zero solution of system (3) is asymptotically stable. The proof of Theorem 1 is now completed.

\section{Boundedness}

To study the boundedness of solutions of (2), we would need to write (2) in the form

$$
\begin{aligned}
x^{\prime}= & y \\
(11) \quad y^{\prime}= & z \\
(12)(p(t) Z)^{\prime}= & -a(t) z-\beta_{2} a(t) z\left(t-r_{2}\right)-b(t) y-\beta_{3} b(t) y\left(t-r_{3}\right) \\
& -c(t) f(x(t))+c(t) \int_{t-\sigma}^{t} f^{\prime}(x(s)) y(s) d s+e(t, x, y, z) .
\end{aligned}
$$

For the next theorem, we impose the following conditions.

$$
|e(t, x, y, z)| \leq h(t)
$$

and

$$
\int_{t_{1}}^{t}|h(s)| d s<D
$$


Theorem 2. If all the assumptions of Theorem 1 and (13)-(14) hold, then there exists a positive constant $N$ such that any solution of (11) satisfies

$$
|x(t)| \leq N,|y(t)| \leq N,|Y(t)| \leq N,|Z(t)| \leq N, \quad \forall t \geq t_{1} \geq 0 .
$$

Proof. Along any solution $(x(t), y(t), Z(t))$ of $(11)$, we have

$$
W_{(11)}^{\prime}=W_{(3)}^{\prime}+\exp \left(-\frac{1}{\omega} \int_{t_{1}}^{t}\left(\left|p^{\prime}(s)\right|+\left|q^{\prime}(s)\right|\right) d s\right)(d y+p(t) Z) e(t, x, y, z)
$$

From (10), we obtain

$$
W_{(11)}^{\prime} \leq K_{1}|h(t)|(|y|+|Z|)
$$

where $K_{1}=\exp \left(\frac{2 k_{1}}{k}\left(p_{1}+q_{1}\right)\right) \max \left\{d, p_{1}\right\}$. Now, the inequality (7), $|y| \leq y^{2}+1$ and $|Z| \leq Z^{2}+1$, lead

$$
\begin{aligned}
W_{(11)}^{\prime} & \leq K_{1}|h(t)|\left(y^{2}+Z^{2}+2\right) \\
& \leq K_{2}|h(t)| W(t)+K_{2}|h(t)|,
\end{aligned}
$$

where $K_{2}=\max \left\{\frac{K_{1}}{k_{0}}, 2 K_{1}\right\}$. Integrating the above estimate from $t_{1}$ to $t$, $t \geq t_{1}=t_{0}+\bar{r}$, one can easily obtain

$$
W(t)-W\left(t_{1}\right) \leq K_{2} \int_{t_{1}}^{t}|h(s)| d s+K_{2} \int_{t_{1}}^{t} W(s)|h(s)| d s .
$$

Thus

$$
W(t) \leq W\left(t_{1}\right)+K_{2} D+K_{2} \int_{t_{1}}^{t} W(s)|h(s)| d s .
$$

Using Gronwall inequality, it follows that

$$
W(t) \leq\left(W\left(t_{1}\right)+K_{2} D\right) \exp \left(K_{2} \int_{t_{1}}^{t}|h(s)| d s\right) \leq D_{1},
$$

where $D_{1}=\left(W\left(t_{1}\right)+K_{2} D\right) \exp \left(K_{2} D\right)$. This result implies that there exists a constant $N$ such that

$$
|x(t)| \leq N,|y(t)| \leq N,|Y(t)| \leq N,|Z(t)| \leq N, \quad \forall t \geq t_{1} .
$$

This completes the proof of Theorem 2 .

\section{Square integrability}

Our next result concerns the square integrability of solutions of equation $(2)$.

Theorem 3. In addition to the assumptions of Theorem 2, if we assume that

$$
\left.J_{5}\right) c_{0} M-\frac{b_{1}}{2}\left(1+\beta_{3}\right)>0 \text {. }
$$


$\left.J_{6}\right) \int_{t_{1}}^{+\infty}\left|a^{\prime}(s)\right| d s<\alpha$.

Then all the solutions of (11) are elements of $L^{2}\left[t_{1},+\infty\right)$.

Proof. Define $U(t)$ as

$$
U(t)=W(t)+\varepsilon \int_{t_{1}}^{t}\left(z^{2}(s)+y^{2}(s)\right) d s, \quad \forall t \geq t_{1},
$$

where $\varepsilon>0$ is a constant to be specified later. By differentiating $U(t)$ along the solution of system (11) and using (10) and (16) we obtain

$$
U_{(11)}^{\prime}(t) \leq\left(\varepsilon-\delta_{1}\right)\left(z^{2}(t)+y^{2}(t)\right)+K_{2}(W(t)+1)|h(t)| .
$$

If we choose $\varepsilon-N<0$, then from (17) we get

$$
U_{(11)}^{\prime}(t) \leq K_{4}|h(t)|,
$$

where $K_{4}=K_{2}\left(D_{1}+1\right)$. Integrating (19) from $t_{1}$ to $t$, and using condition (14) of Theorem 2 we obtain

$$
U(t)-U\left(t_{1}\right)=\int_{t_{1}}^{t} U_{(11)}^{\prime}(s) d s \leq K_{4} D .
$$

Using equality $U\left(t_{1}\right)=W\left(t_{1}\right)$ we get

$$
U(t) \leq K_{4} D+W\left(t_{1}\right) .
$$

We can conclude by (18) that

$$
\int_{t_{1}}^{t}\left(y^{2}(s)+z^{2}(s)\right) d s<\frac{K_{4} D+W\left(t_{1}\right)}{\varepsilon},
$$

which imply the existence of positive constants $\zeta_{1}$ and $\zeta_{2}$ such that

$$
\int_{t_{1}}^{t} x^{\prime \prime 2}(s) d s=\int_{t_{1}}^{t} z^{2}(s) d s \leq \zeta_{2}
$$

and

By the fact that

$$
\int_{t_{1}}^{t} x^{\prime 2}(s) d s=\int_{t_{1}}^{t} y^{2}(s) d s \leq \zeta_{1}
$$

$$
\int_{t_{1}}^{t} x^{\prime 2}\left(s-r_{1}\right) d s=\int_{t_{0}+\bar{r}-r_{1}}^{t-r_{1}} x^{\prime 2}(u) d u \leq \int_{t_{0}+\bar{r}-r_{1}}^{t_{1}} x^{\prime 2}(u) d u+\zeta_{1} \leq \kappa_{1}+\zeta_{1},
$$

and

$$
\int_{t_{1}}^{t} x^{\prime \prime 2}\left(s-r_{1}\right) d s=\int_{t_{0}+\bar{r}-r_{1}}^{t-r_{1}} x^{\prime \prime 2}(u) d u \leq \int_{t_{0}+\bar{r}-r_{1}}^{t_{1}} x^{\prime \prime 2}(u) d u+\zeta_{2} \leq \kappa_{2}+\zeta_{2},
$$

we deduce by using the estimate $2 u v \leq u^{2}+v^{2}$ and $\left(\mathrm{J}_{0}\right),\left(\mathrm{J}_{1}\right)$, that

$$
\int_{t_{1}}^{t} Z^{2}(s) d s=\int_{t_{1}}^{t}\left(q^{\prime}(s)\left(x^{\prime}(s)+\beta_{1} x^{\prime}\left(s-r_{1}\right)\right)\right.
$$




$$
\begin{aligned}
& \left.+q(s)\left(x^{\prime \prime}(s)+\beta_{1} x^{\prime \prime}\left(s-r_{1}\right)\right)\right)^{2} d s \\
& \leq\left(1+\beta_{1}\right)\left(L+q_{1}\right) \int_{t_{1}}^{t}\left(L\left(x^{\prime 2}(s)+\beta_{1} x^{\prime 2}\left(s-r_{1}\right)\right)\right. \\
& \left.+q_{1}\left(x^{\prime \prime 2}(s)+\beta_{1} x^{\prime \prime 2}\left(s-r_{1}\right)\right)\right) d s \\
& \leq\left(1+\beta_{1}\right)\left(L+q_{1}\right)\left(L\left(\zeta_{1}+\beta_{1}\left(\kappa_{1}+\zeta_{1}\right)\right)\right. \\
& \left.+q_{1}\left(\zeta_{2}+\beta_{1}\left(\kappa_{2}+\zeta_{2}\right)\right)\right)=\zeta_{3} .
\end{aligned}
$$

We assert that $\int_{t_{1}}^{t} x^{2}(s) d s<\infty$, to prove this we multiply (2) by $x(t-\sigma)$, we obtain

$$
\begin{aligned}
& x(t-\sigma)\left[p(t)\left(q^{\prime}(t)\left(x^{\prime}(t)+\beta_{1} x^{\prime}\left(t-r_{1}\right)\right)+q(t)\left(x^{\prime \prime}(t)+\beta_{1} x^{\prime \prime}\left(t-r_{1}\right)\right)\right)\right]^{\prime} \\
& +a(t) x(t-\sigma)\left(x^{\prime \prime}(t)+\beta_{2} x^{\prime \prime}\left(t-r_{2}\right)\right)+b(t) x(t-\sigma)\left(x^{\prime}(t)+\beta_{3} x^{\prime}\left(t-r_{3}\right)\right) \\
& (20) \quad+c(t) x(t-\sigma) f(x(t-\sigma))=x(t-\sigma) e\left(t, x, x^{\prime}, x^{\prime \prime}\right) .
\end{aligned}
$$

Integrating (20) from $t_{1}$ to $t$, we have

$$
\int_{t_{1}}^{t} c(s) x(s-\sigma) f(x(s-\sigma)) d s=\Delta_{1}(t)+\Delta_{2}(t)+\Delta_{3}(t),
$$

where

$$
\begin{aligned}
\Delta_{1}(t)= & -\int_{t_{1}}^{t} x(s-\sigma)\left(p ( s ) \left(q^{\prime}(s)\left(x^{\prime}(s)+\beta_{1} x^{\prime}\left(s-r_{1}\right)\right)\right.\right. \\
& \left.\left.+q(s)\left(x^{\prime \prime}(s)+\beta_{1} x^{\prime \prime}\left(s-r_{1}\right)\right)\right)\right)^{\prime} d s \\
\Delta_{2}(t)= & -\int_{t_{1}}^{t}\left(a(s) x(s-\sigma)\left(x^{\prime \prime}(s)+\beta_{2} x^{\prime \prime}\left(s-r_{2}\right)\right)\right. \\
& \left.+b(s) x(s-\sigma)\left(x^{\prime}(s)+\beta_{3} x^{\prime}\left(s-r_{3}\right)\right)\right) d s \\
\Delta_{3}(t)= & \int_{t_{1}}^{t} x(s-\sigma) e\left(s, x(s), x^{\prime}(s), x^{\prime \prime}(s)\right) d s .
\end{aligned}
$$

Integrating by parts and using the estimate $2 u v \leq u^{2}+v^{2}$ we obtain

$$
\begin{aligned}
\Delta_{1}(t)= & M_{1}(t)-M_{1}\left(t_{1}\right)+\int_{t_{1}}^{t}\left(p(s) q^{\prime}(s) x^{\prime}(s-\sigma)\left(x^{\prime}(s)+\beta_{1} x^{\prime}\left(s-r_{1}\right)\right)\right. \\
& \left.+p(s) q(s) x^{\prime}(s-\sigma)\left(x^{\prime \prime}(s)+\beta_{1} x^{\prime \prime}\left(s-r_{1}\right)\right)\right) d s
\end{aligned}
$$




$$
\begin{array}{ll}
\leq & \left|M_{1}(t)-M_{1}\left(t_{1}\right)\right|+\int_{t_{1}}^{t}\left(\left|p(s) q^{\prime}(s) x^{\prime}(s-\sigma)\left(x^{\prime}(s)+\beta_{1} x^{\prime}\left(s-r_{1}\right)\right)\right|\right. \\
& \left.+\left|p(s) q(s) x^{\prime}(s-\sigma)\left(x^{\prime \prime}(s)+\beta_{1} x^{\prime \prime}\left(s-r_{1}\right)\right)\right|\right) d s \\
\leq & \left|M_{1}(t)-M_{1}\left(t_{1}\right)\right|+\frac{p_{1} L}{2} \int_{t_{1}}^{t}\left(\left(1+\beta_{1}\right) x^{\prime 2}(s-\sigma)+x^{\prime 2}(s)\right. \\
& \left.+\beta_{1} x^{\prime 2}\left(s-r_{1}\right)\right) d s+\frac{p_{1} q_{1}}{2} \int_{t_{1}}^{t}\left(\left(1+\beta_{1}\right) x^{\prime 2}(s-\sigma)+x^{\prime \prime 2}(s)\right. \\
& \left.+\beta_{1} x^{\prime \prime 2}\left(s-r_{1}\right)\right) d s,
\end{array}
$$

where

$$
M_{1}(t)=-p(t) x(t-\sigma) Z(t) .
$$

We remark by $\left(\mathrm{J}_{0}\right)$ and the inequalities (15) that

$$
\left|M_{1}(t)-M_{1}\left(t_{1}\right)\right| \leq p_{1} N^{2}+\left|M_{1}\left(t_{1}\right)\right|, \text { for all } t \geq t_{1},
$$

and

$$
\int_{t_{1}}^{t} x^{\prime 2}(s-\sigma) d s=\int_{t_{0}+\bar{r}-\sigma}^{t-\sigma} x^{\prime 2}(u) d u \leq \int_{t_{0}+\bar{r}-\sigma}^{t_{1}} x^{2}(u) d u+\zeta_{1} \leq \kappa_{3}+\zeta_{1},
$$

thus

$$
\begin{aligned}
\Delta_{1}(t) & \leq p_{1} N^{2}+\left|M_{1}\left(t_{1}\right)\right|+\frac{p_{1}}{2}\left(\left(1+\beta_{1}\right)\left(\left(L+q_{1}\right)\left(\kappa_{3}+\zeta_{1}\right)+L \zeta_{1}+q_{1} \zeta_{2}\right)\right. \\
& \left.+\beta_{1}\left(L \kappa_{1}+q_{1} \kappa_{2}\right)\right)=l_{1} .
\end{aligned}
$$

Similarly, we have

$$
\begin{aligned}
\Delta_{2}(t)= & -\int_{t_{1}}^{t}\left(a(s) x(s-\sigma)\left(x^{\prime \prime}(s)+\beta_{2} x^{\prime \prime}\left(s-r_{2}\right)\right)\right. \\
& \left.+b(s) x(s-\sigma)\left(x^{\prime}(s)+\beta_{3} x^{\prime}\left(s-r_{3}\right)\right)\right) d s \\
= & -a(t) x(t-\sigma)\left(x^{\prime}(t)+\beta_{2} x^{\prime}\left(t-r_{2}\right)\right)+M_{2}\left(t_{1}\right) \\
& +a(t) \int_{t_{1}}^{t} x^{\prime}(s-\sigma)\left(x^{\prime}(s)+\beta_{2} x^{\prime}\left(s-r_{2}\right)\right) d s \\
& +\int_{t_{1}}^{t} a^{\prime}(s) x(s-\sigma)\left(x^{\prime}(s)+\beta_{2} x^{\prime}\left(s-r_{2}\right)\right) d s \\
& -\int_{t_{1}}^{t} a^{\prime}(s)\left[\int_{t_{1}}^{s} x^{\prime}(u-\sigma)\left(x^{\prime}(u)+\beta_{2} x^{\prime}\left(u-r_{2}\right)\right) d u\right] d s \\
& -\int_{t_{1}}^{t} b(s) x(s-\sigma)\left(x^{\prime}(s)+\beta_{3} x^{\prime}\left(s-r_{3}\right)\right) d s,
\end{aligned}
$$


where $M_{2}\left(t_{1}\right)=a\left(t_{1}\right) x\left(t_{1}-\sigma\right)\left(x^{\prime}\left(t_{1}\right)+\beta_{2} x^{\prime}\left(t_{1}-r_{2}\right)\right)$. Using

$$
\int_{t_{1}}^{t} x^{\prime 2}\left(s-r_{2}\right) d s=\int_{t_{0}+\bar{r}-r_{2}}^{t-r_{2}} x^{\prime 2}(u) d u \leq \int_{t_{0}+\bar{r}-r_{2}}^{t_{1}} x^{\prime 2}(u) d u+\zeta_{1} \leq \kappa_{4}+\zeta_{1},
$$

and

$$
\int_{t_{1}}^{t} x^{\prime 2}\left(s-r_{3}\right) d s=\int_{t_{0}+\bar{r}-r_{3}}^{t-r_{3}} x^{\prime 2}(u) d u \leq \int_{t_{0}+\bar{r}-r_{3}}^{t_{1}} x^{\prime 2}(u) d u+\zeta_{1} \leq \kappa_{5}+\zeta_{1},
$$

then

$$
\begin{aligned}
\Delta_{2}(t) & \leq a_{1}\left(\left(N^{2}+\zeta_{1}+\frac{\kappa_{3}}{2}\right)\left(1+\beta_{2}\right)+\beta_{2} \frac{\kappa_{4}}{2}\right)+\left|M_{2}\left(t_{1}\right)\right| \\
& +\frac{b_{1}}{2}\left(1+\beta_{3}\right) \int_{t_{1}}^{t} x^{2}(s-\sigma) d s+\frac{b_{1}}{2}\left(\zeta_{1}\left(1+\beta_{3}\right)+\beta_{3} \kappa_{5}\right) \\
& +\int_{t_{1}}^{t}\left(\left|a^{\prime}(s)\right||x(s-\sigma)|\left(\left|x^{\prime}(s)\right|+\beta_{2}\left|x^{\prime}\left(s-r_{2}\right)\right|\right)\right. \\
& \left.+\left|a^{\prime}(s)\right|\left[\int_{t_{1}}^{s}\left|x^{\prime}(u-\sigma)\right|\left(\left|x^{\prime}(u)\right|+\beta_{2}\left|x^{\prime}\left(u-r_{2}\right)\right|\right) d u\right]\right) d s \\
& \leq\left(\left(N^{2}+\zeta_{1}+\frac{\kappa_{3}}{2}\right)\left(1+\beta_{2}\right)+\beta_{2} \frac{\kappa_{4}}{2}\right)\left(a_{1}+\int_{t_{1}}^{t}\left|a^{\prime}(s)\right| d s\right) \\
& +\left|M_{2}\left(t_{1}\right)\right|+\frac{b_{1}}{2}\left(\zeta_{1}\left(1+\beta_{3}\right)+\beta_{3} \kappa_{5}\right)+\frac{b_{1}}{2}\left(1+\beta_{3}\right) \int_{t_{1}}^{t} x^{2}(s-\sigma) d s .
\end{aligned}
$$

Next

$$
\begin{aligned}
\Delta_{3}(t) & \leq \int_{t_{1}}^{t}|x(s-\sigma)|\left|e\left(s, x(s), x^{\prime}(s), x^{\prime \prime}(s)\right)\right| d s \\
& \leq N \int_{t_{1}}^{t}|h(s)| d s \\
& \leq N D .
\end{aligned}
$$

By $(21)$ and conditions $\left(\mathrm{J}_{0}\right),\left(\mathrm{J}_{2}\right)$, we obtain

$$
\begin{aligned}
c_{0} M \int_{t_{1}}^{t} x^{2}(s-\sigma) d s & \leq \int_{t_{1}}^{t} c(s) x(s-\sigma) f(x(s-\sigma)) d s \\
& \leq K+\frac{b_{1}}{2}\left(1+\beta_{3}\right) \int_{t_{1}}^{t} x^{2}(s-\sigma) d s,
\end{aligned}
$$

where

$$
\begin{aligned}
K & =l_{1}+\left(\left(N^{2}+\zeta_{1}+\frac{\kappa_{3}}{2}\right)\left(1+\beta_{2}\right)+\beta_{2} \frac{\kappa_{4}}{2}\right)\left(a_{1}+\alpha\right) \\
& +\left|M_{2}\left(t_{1}\right)\right|+\frac{b_{1}}{2}\left(\zeta_{1}\left(1+\beta_{3}\right)+\beta_{3} \kappa_{5}\right)+N D .
\end{aligned}
$$


Then

$$
\left[c_{0} M-\frac{b_{1}}{2}\left(1+\beta_{3}\right)\right] \int_{t_{1}}^{t} x^{2}(s-\sigma) d s \leq K .
$$

From condition $\left(\mathrm{J}_{5}\right)$, it follows that $\int_{t_{1}}^{t} x^{2}(s-\sigma) d s<\infty$, hence $\int_{t_{1}}^{+\infty} x^{2}(s) d s<\infty$. This fact completes the proof of theorem.

\section{EXAMPLE}

We consider the following third order non-autonomous delay neutral differential equation

$$
\begin{aligned}
& {\left[\left(\frac{1}{10+t^{2}}+\frac{3}{10}\right)\left(\left(\frac{1}{20+t^{2}}+\frac{4}{10}\right)\left(x(t)+\beta_{1} x\left(t-r_{1}\right)\right)^{\prime}\right)^{\prime}\right]^{\prime}} \\
& +\left(\frac{1}{2 \pi} \arctan t+\frac{25}{4}\right)\left(x^{\prime \prime}(t)+\beta_{2} x^{\prime \prime}\left(t-r_{2}\right)\right) \\
& +\left(\frac{1}{1+t^{2}}+5\right)\left(x^{\prime}(t)+\beta_{3} x^{\prime}\left(t-r_{3}\right)\right)+\left(\frac{1}{4+t^{2}}+5\right) \\
& {\left[\frac{7}{10}\left(x(t-\sigma)+\frac{x(t-\sigma)}{1+x^{2}(t-\sigma)}\right)\right]=\frac{\sin t}{1+t^{2}+|x|+\left|x^{\prime}\right|+\left|x^{\prime \prime}\right|} .}
\end{aligned}
$$

Now, it is easy to see that for all $t \geq t_{1}$,

$$
\begin{aligned}
& \frac{3}{10}=p_{0} \leq p(t)=\frac{1}{10+t^{2}}+\frac{3}{10} \leq \frac{4}{10}=p_{1}, \\
& -L=-0.021=-\frac{3 \sqrt{3}}{80 \sqrt{10}} \leq p^{\prime}(t)=\frac{-2 t}{\left(10+t^{2}\right)^{2}} \leq 0, \\
& \frac{4}{10}=q_{0} \leq q(t)=\frac{1}{20+t^{2}}+\frac{4}{10} \leq \frac{9}{20}=q_{1}, \\
& -L=-0,021=-\frac{3 \sqrt{3}}{80 \sqrt{10}} \leq q^{\prime}(t)=\frac{-2 t}{\left(20+t^{2}\right)^{2}} \leq 0, \\
& 6=a_{0} \leq a(t)=\frac{1}{2 \pi} \arctan t+\frac{25}{4} \leq \frac{26}{4}=a_{1}, \\
& a^{\prime}(t)=\frac{1}{2 \pi\left(1+t^{2}\right)} \leq \frac{1}{2 \pi}, \\
& 5=c_{0} \leq c(t)=\frac{1}{4+t^{2}}+5 \leq b(t)=\frac{1}{1+t^{2}}+5 \leq 6=b_{1}, \\
& \frac{7}{10}=M \leq \frac{f(x)}{x}=\frac{7}{10}\left(1+\frac{1}{1+x^{2}}\right) \text { with } x \neq 0, \text { and }\left|f^{\prime}(x)\right| \leq \frac{7}{5}=\delta,
\end{aligned}
$$




$$
\begin{aligned}
& \frac{\sin t}{1+t^{2}+|x|+|y|+|z|} \leq \frac{1}{1+t^{2}}=h(t), \text { and } \\
& \int_{t_{1}}^{t}|h(s)| d s<\frac{\pi}{2}=D \text { and } \int_{t_{1}}^{t}\left|a^{\prime}(s)\right| d s<\frac{1}{4}=\alpha .
\end{aligned}
$$

It is straightforward to verify for all $t \geq t_{1}$, that

$$
\begin{aligned}
& 2 \delta p_{1} q_{1}=0.504<d<6=a_{0} \\
& d a^{\prime}(t)+2 c_{0}\left(p_{1} q_{1} \delta\left(1+\frac{\beta_{1}}{2}\right)-d\right)+\left(\beta_{2} a_{1}+\beta_{3} b_{1}\right)\left(d+L \beta_{1} p_{1}\right) \\
& +\beta_{1} p_{1}\left(b_{1} q_{1} \delta+b_{1} L+b_{1} q_{1}\left(1+\beta_{1}\right)+L\left(a_{1}-d\right)\right)+b_{1} \beta_{3} n_{1} \leq-1,7<0 \\
& \text { for } d=0.6 \text { and } \beta_{i}=\frac{1}{10} \\
& p_{0} q_{0}\left(2-\beta_{1}\right)\left(d-a_{0}\right)+p_{1} q_{1}\left(\beta_{1} b_{1}+\beta_{2} a_{1}+\beta_{3} b_{1}\right)\left(1+\beta_{1}\right) \\
& +p_{1}\left(a_{1}-d\right)\left(\beta_{1} q_{1}+L\left(1+\beta_{1}\right)\right)+a_{1} \beta_{2} n_{1} \leq-0.17<0 \\
& c_{0} M-\frac{b_{1}}{2}\left(1+\beta_{3}\right)=\frac{7}{2}-3\left(1+\frac{1}{10}\right)=\frac{1}{5}>0 .
\end{aligned}
$$

All the assumptions of Theorem 3 are satisfied, we can conclude that every solution of (22) are bounded and elements of $L^{2}\left[t_{1},+\infty\right)$.

\section{REFERENCES}

[1] O. Arino, M. L. Hbid, and E. Ait Dads, Delay Differential Equations and Applications, NATO sciences series, Berlin, Springer, 2006.

[2] T. Ayhan, C. Tunç, On the global existence and boundedness of solutions of nonlinear vector differential equations of third order, Applications and Applied Mathematics: An International Journal (AAM), 11 (1) (2016), 152-161.

[3] B. Baculíková, J. Džurina, On the asymptotic behavior of a class of third order nonlinear neutral differential equations, Central European Journal of Mathematics, 8 (2010), 1091-1103.

[4] B. Mihalíková and E. Kostiková, Boundedness And Oscillation Of Third Order Neutral Differential Equations, Tatra Mountains Mathematical Publications, 43 (2009), 137-144.

[5] P. Das, N. Misra, A necessary and sufficient condition for the solution of a functional differential equation to be oscillatory or tend to zero, Journal of Mathematical Analysis and Applications, 204 (1997), 78-87.

[6] B. Dorociaková, Some nonoscillatory properties of third order differential equations of neutral type, Tatra Mountains Mathematical Publications, 38 (2007), 71-76.

[7] Z. Došlá, P. Liška, Oscillation of third-order nonlinear neutral differential equations, Applied Mathematics Letters, 56 (2016), 42-48.

[8] Z. Došla, P. Liška, Comparison Theorems For Third-Order Neutral Differential Equations, Electronic Journal of Differential Equations, Vol. 38 (2016), 1-13. 
[9] L. E. El'sgol'ts, Introduction to the theory of differential equations with deviating arguments, Translated from the Russian by Robert J. McLaughlin Holden-Day, Inc., San Francisco, California - London - Amsterdam, 1966.

[10] J. R. Graef, D. Beldjerd, M. Remili, On stability, ultimate boundedness, and existence of periodic solutions of certain third order differential equations with delay, PanAmerican Mathematical Journal, 25 (2015), 82-94.

[11] J. R. Graef, L. D. Oudjedi, M. Remili, Stability and square integrability of solutions of nonlinear third order differential equations, Dynamics of Continuous, Discrete and Impulsive Systems Series A: Mathematical Analysis, 22 (2015) 313-324.

[12] J. R. Graef, L. D. Oudjedi, M. Remili, Stability and Square Integrability of solutions to third order neutral delay differential equations, Tatra Mountains Mathematical Publications, 71 (2018), 81-97.

[13] J. K. Hale, Theory of Functional Differential Equations, Springer-Verlag, New York, 1977.

[14] J. K. Hale, S. M. V. Lunel, Introductiont to Functional-Differential Equations, Springer-Verlag, New York, 1993.

[15] M. R. S. Kulenovic, G. Ladas, A. Meimaridou, Stability of solutions of linear delay differential equations, Proceedings of the American Mathematical Society, 100 (1987), 433-441.

[16] A. M. Mahmoud, On the Asymptotic Stability of Solutions for a Certain Nonautonomous Third-order Delay Differential Equation, British Journal of Mathematics and Computer Science, 16 (3) (2016), 1-12.

[17] M. O. Omeike, New results on the stability of solution of some non-autonomous delay differential equations of the third order, Differential Equations and Control Processes, 2010 (1) (2010), 18-29.

[18] L. D. Oudjedi, D. Beldjerd, M. Remili, On the Stability of Solutions for non- autonomous delay differential equations of third-order, Differential Equations and Control Processes, 2014 (1) (2014), 22-34.

[19] M. Remili, D. Beldjerd, A boundedness and stability results for a kind of third order delay differential equations, Applications and Applied Mathematics, 10 (2) (2015), $772-782$.

[20] M. Remili, D. Beldjerd, On the asymptotic behavior of the solutions of third order delay differential equations, Rendiconti del Circolo Matematico di Palermo Series 2, 63 (3) (2014), 447-455.

[21] M. Remili, D. Beldjerd, On ultimate boundedness and existence of periodic solutions of kind of third order delay differential equations, Acta Universitatis Matthiae Belii, series Mathematics, 2016 (2016), 1-15.

[22] M. Remili, D. Beldjerd, Stability and ultimate boundedness of solutions of some third order differential equations with delay, Journal of the Association of Arab Universities for Basic and Applied Sciences, 23 (2017), 90-95.

[23] M. Remili, L. D. Oudjedi, Boundedness and stability in third order nonlinear differential equations with bounded delay, Analele Universităţii Oradea Fasc. Matematica, XXIII (1) (2016), 135-143.

[24] M. Remili, L. D. Oudjedi, Boundedness and stability in third order nonlinear differential equations with multiple deviating arguments, Archivum Mathematicum, 52 (2) (2016), 79-90. 
[25] M. Remili, L. D. Oudjedi, Stability and Boundedness of the Solutions of Non Autonomous Third Order Differential Equations with Delay, Acta Univ. Palacki. Olomuc., Fac. rer. nat., Mathematica 53 (2) (2014), 139-147.

[26] M. Remili, L. D. Oudjedi, Stability of the solutions of nonlinear third order differential equations with multiple deviating arguments, Acta Universitatis Sapientiae, Mathematica, 8 (1), (2016) 150-165.

[27] M. Remili, L. D. Oudjedi, On asymptotic stability of solutions to third order nonlinear delay differential equation, Filomat, 30 (12) (2016), 3217-3226.

[28] M. Remili, L. D. Oudjedi, and Beldjerd, D., On the qualitative behaviors of solutions to a kind of nonlinear third order differential equation with delay, Communications in Applied Analysis, 20 (2016), 53-64.

[29] M. Remili, L. D. Oudjedi, Uniform Stability and Boundedness of a Kind of Third Order Delay Differential Equations, Bulletin of Computational Applied Mathematics, 2 (1) (2014), 25-35.

[30] M. Remili, L. D. Oudjedi, Uniform ultimate boundedness and asymptotic behaviour of third order nonlinear delay differential equation, Afrika Matematika, 27 (7) (2016), $1227-1237$.

[31] Y.-Z. Tian, Y.-L. Cai, Y.-L. Fu, T.-X. Li, Oscillation and asymptotic behavior of third-order neutral differential equations with distributed deviating arguments. Advances in Difference Equations, 267 (2015), 14 pages.

[32] T.-X. Li, C.-H. Zhang, G.-J. Xing, Oscillation of third-order neutral delay differential equations, Abstract and Applied Analysis, 2012 (2012), 11 pages.

[33] J. Yu, Z. Wang, C. Qian, Oscillation of neutral delay differential equation, Bulletin of the Australian Mathematical Society, 45 (1992), 195-200.

[34] Yu Jianshe, Asymptotic Stability For A Class Of Nonautonomous Neutral Differential Equations, Chinese Annals of Mathematics, Series B, 18 (4) (1997), 449-456.

\section{Moussadek Remili}

Department of Mathematics

University of Oran 1 Ahmed Ben Bella

31000 ORAN

Algeria

E-mail address: remilimous@gmail.com

LINDA D. OUDJEDI

Department of Mathematics

University of Oran 1 Ahmed Ben Bella

31000 ORAN

Algeria

E-mail address: oudjedi@yahoo.fr 